\title{
Clinic serum levels of Plin5 is therapeutic target of spinal cord injury and Plin5 reduced inflammation in spinal cord injury via silent information regulator 1 dependent inhibition of NLRP3 inflammasome
}

\author{
Binqiang $\mathrm{WU}^{1+}$, Xiao $\mathrm{LIANG}^{1+}$, Feng $\mathrm{ZHAO}^{1}$, Wei FAN ${ }^{1}$, Chunjiang $\mathrm{LI}^{1}$, Bin $\mathrm{ZHAO}^{1 \star}$ (D), Jie REN ${ }^{1 \star}$
}

\begin{abstract}
The study investigated that clinic significance and molecular mechanism of Plin5 in spinal cord injury (SCI). Serum levels of Plin 5 was down-regulated and there is a negative correlation between Plin 5 and IL- $1 \beta$ levels in patients with SCI,. Human Plin5 protein could reduce inflammation, and prevented spinal cord injury in rat model of SCI. Over-expression of Plin 5 reduced inflammation divisors via activation of SIRT1 and suppression of NLRP3 in vitro model of SCI; down-regulation of Plin5 promoted inflammation divisors via inactivation of SIRT1 and induction of NLRP3 in vitro model of SCI. SIRT1 is important targets of Plin5 in inflammation divisors of SCI. The inhibition of SIRT1 reduced the effects of Plin5 on inflammation divisors of SCI. The activation of NLRP3 also reduced the effects of Plin5 on inflammation divisors of SCI. We concluded that clinic Serum levels of Plin5 is therapeutic target of SCI and Plin 5 reduced inflammation in SCI via SIRT1 dependent suppression of NLRP3 Inflammasome.
\end{abstract}

Keywords: Plin5; SIRT1; NLRP3; spinal cord injury.

Practical Application: Clinic significance and molecular mechanism of Plin5 in spinal cord injury.

\section{Introduction}

Spinal cord injury (SCI) can be divided into primary injury and secondary injury according to the molecular mechanism of pathogenesis (Gensel \& Zhang, 2015). Primary SCI is caused by the direct or indirect action of initial external force on the spinal cord, while secondary SCI is caused by destructive lesions in the initially complete tissues surrounding primary SCI following a sequence of physiological and biochemical mechanisms, including apoptosis, oxidative stress and inflammatory response, further aggravating the degree of damage and expanding the scope of damage (Kjell \& Olson, 2016; Järve et al., 2020). The post-SCI inflammatory response is relatively complex, involving both nerves and immune systems, and the dynamic role of various factors, all of which become important events that constitute secondary SCI (Kornhaber et al., 2018; Hoey \& Hubscher, 2020).

SCI will not only cause cellular oxidative stress response, cell necrosis, and apoptosis, but also give rise to the local secretion of a great many inflammatory mediators, resulting in immune inflammatory response (Ma et al., 2020; Liu et al. 2020). The inflammatory response in this secondary injury plays an important role in the further recovery of science and technology (Yu \& Qian, 2020).

Silent information regulator 1 (SIRT1) related enzyme is a highly conserved $\mathrm{NAD}^{+}$-dependent protein deacetylase (Wang et al., 2018). There are seven homologous genes of Sir2 in humans: namely SIRT1-7 (Wang et al., 2018). Among them,
SIRT1 has the highest degree of homology and has become a research hotspot in recent years (Lu et al., 2019; Ding et al., 2021; Wang et al., 2021). SIRT1 protein has been found to exert anti-inflammatory and anti-oxidative stress effects, which can attenuate cell damage. The functions of SIRT1 is relatively complicated, which can interact with proteins in a variety of signal transduction pathways (Wang et al., 2019). To be specific, it can deacetylate histone, lysine residue, and transcription factors in the organism, and is involved in neuroprotection, cell aging and apoptosis, glycolipid metabolism, insulin secretion, inflammatory oxidative stress response, angiogenesis, thereby regulating gene functions (Yu et al., 2019a).

NLRP3 is a member of the NLRs family and is the core component of the inflammasome (Pu et al., 2019). It contains three domains and its $\mathrm{C}$-terminus is a leucine-rich repeat domain, whose main function is to identify danger-associated molecular patterns or pathogen-associated molecular patterns, also playing an important role in self-regulation and proteinprotein interaction (Jiang et al., 2019). TLR can induce the activation of NF- $\kappa \mathrm{B}$, and is involved in the regulation of NLRP3 transcription by combining with the NF- $\kappa B$-related sites in the NLRRP3 promoter (Mohammed et al., 2020). Relevant studies have also confirmed that NF- $\mathrm{kB}$ signaling pathway can regulate the protein expression of NLRP inflammasome, IL-18 precursor and IL- $1 \beta$ precursor in peripheral immune cells (Jiang et al., 2019; Zhang et al., 2020b; Su et al., 2020). The study investigated 
that clinic significance and molecular mechanism of Plin5 on inflammation of SCI.

\section{Materials and methods}

\subsection{Animals model}

All experiments were performed in accordance with National Institutes of Health guidelines and were approved by Institutional Animal Care and Use Committees at the University of California, San Francisco. SD rats were housed at $22-23{ }^{\circ} \mathrm{C}$ and $55-60 \%$ humidity with ad libitum access to food and water.

All rats were administered with $35 \mathrm{mg} / \mathrm{kg}$ pentobarbital sodium and fixed on the operating table and $\mathrm{T} 9$ spinous processes. Subcutaneous tissues were incised along the posterior median line. T8-T9 spinous processes and lamina were exposed and processes and lamina were removed, right side of the spinal cord was cut and right hind limb was considered to indicate a successful model of SCI. All rats were random distributed into control and SCI model groups.

\subsection{Histological analyses}

After treatment, spinal cord tissues were fixed in $4 \%$ formalin for $24 \mathrm{~h}$ and tissue were embedded in paraffin. Tissue samples were cut $5 \mu \mathrm{m}$ of section and it was stained with HE sassy. Histological analyses were observed via an optical microscope (BX-42, Olympus Corporation, Tokyo, Japan).

\subsection{Cell culture and treatment}

Nerve cell line PC12 cell was purchased from Shanghai Cell Bank of Chinese Academy of Sciences (Shanghai, China) and cultured in Dulbecco's modified Eagle's medium (DMEM) with $10 \%$ fetal bovine serum (FBS) at $37^{\circ} \mathrm{C}$ in $5 \%$ CO2. PC12 cell were transfected with Plin5 plasmid, siPlin5 plasmid, NLRP3 plasmid or negative mimics using Lipofectamine 2000. PC12 cell was induced with $100 \mathrm{ng} / \mathrm{mL}$ LPS for $4 \mathrm{~h}$ and then cultured with ATP $(1 \mathrm{nM})$ for $1 \mathrm{~h}$ after transfection at $48 \mathrm{~h}$.

\subsection{Reverse transcription-quantitative polymerase chain reaction ( $R T-q P C R)$ and Gene chip}

Total RNA were isolated using Trizol reagent (Invitrogen, CA, USA) and TaqMan Reverse Transcription kit (Life Technologies) was then used to synthesize cDNA. RT-qPCR was performed using SYBR-Green Universal qPCR Master Mix (Bio-Rad, Hercules, CA, USA). The RNA quality and quantity were measured by Agilent Bioanalyzer 2100 (Agilent technologies, Santa Clara, CA, USA). The sign were performed by Agilent Microarray Scanner (Agilent technologies, Santa Clara, CA, USA).

\subsection{Measurement of inflammation and oxidative stress}

ROS, MDA, SOD, CAT and GSH-PX, NF-KB p65, TNF- $\alpha$, IL- $1 \beta$ and IL- 6 levels were measured using ELISA kits (Nanjing Jiancheng Biological Engineering Research Institute Co. LTD). The optical density was measured using a Multiskan FC enzyme immunoassay analyzer (Thermo Fisher Scientifc, Waltham, MA, USA) at $450 \mathrm{~nm}$.

\subsection{Protein isolation and western blotting}

Samples were lysed with an ice-cold RIPA buffer (Beyotime Institute of Biotechnology) and protein was quantified using BCA assay (Beyotime Institute of Biotechnology). Equal amount of proteins of the samples were loaded onto the gel and transferred onto a polyvinylidene difluoride membrane. Membrane was blocked with $5 \%$ non-fat in TBST for $1 \mathrm{~h}$ at $37^{\circ} \mathrm{C}$ and incubated with Plin5, SIRT1, NLRP3 and GAPDH followed by recognition with corresponsive secondary antibody. Membrane was washed with TBST and visualizated with an ECL kit (Pierce Chemical Co.). and was analyzed by Image-Pro Plus 6.0 software.

\subsection{Statistical analysis}

The data are presented as mean \pm SEM used GraphPad prism 8 for statistical analysis. $\mathrm{P} \leq 0.05$ was statistically significant used student t-test or two-way ANOVA for statistical analysis when appropriate.

\section{Results}

\subsection{Serum levels of Plin5 in patient with SCI and Plin5 prevented SCI}

To determine whether Plin5 had clinical significance of SCI and the changes of Plin5 expression in response to SCI were analyzed by PCR. For the in vivo study, Plin $5 \mathrm{mRNA}$ expression was down-regulated (Figure 1A). In addition, as a control group, In mice of SCI, human Plin5 protein reduced the water content of spinal cord, increased BBB score and inhibited serum NF-KB p65, TNF- $\alpha$, IL- $1 \beta$ and IL-6 levels (Figure 1B-1H).

\subsection{Plin5 regulated inflammation of SCI}

We next assessed the effects of Plin5 in SCI and overexpression of Plin 5 in vitro model was used in this study. There was increase of Plin5 expression and over-expression of Plin5 reduced NF-KB p65, TNF- $\alpha$, IL-1 $\beta$ and IL-6 levels in vitro model (Figure 2A-2E). The inhibition of Plin5 expression in vitro model was observed and down-regulation of Plin5 increased NF-KB p65, TNF- $\alpha$, IL- $1 \beta$ and IL-6 levels in vitro model (Figure 2F-2J).

\subsection{Plin5 regulated ROS-induced oxidative stress of SCI}

Over-expression of Plin5 reduced ROS production levels and MDA activity level, and increased SOD, CAT and GSH-PX activity levels in vitro model (Figure 3A-3F). The down-regulation of Plin5 promoted ROS production levels and MDA activity level, and reduced SOD, CAT and GSH-PX activity levels in vitro model (Figure 3G-3L).

\subsection{SIRT1 is control targets of anti-inflammation effects of Plin5 in SCI by NLRP3}

To critically determine the mechanism of Plin5 in SCI, gene chip was used the Plin5 regulated gene. Plin5 regulation gene was showed at Figure 4A, and volcano figure showed that a great many regulation gene and SIRT1/NLRP3 were discovered in SCI (Figure 4B). Over-expression of Plin5 induced SIRT1 protein expression and suppressed NLRP3 protein expression 

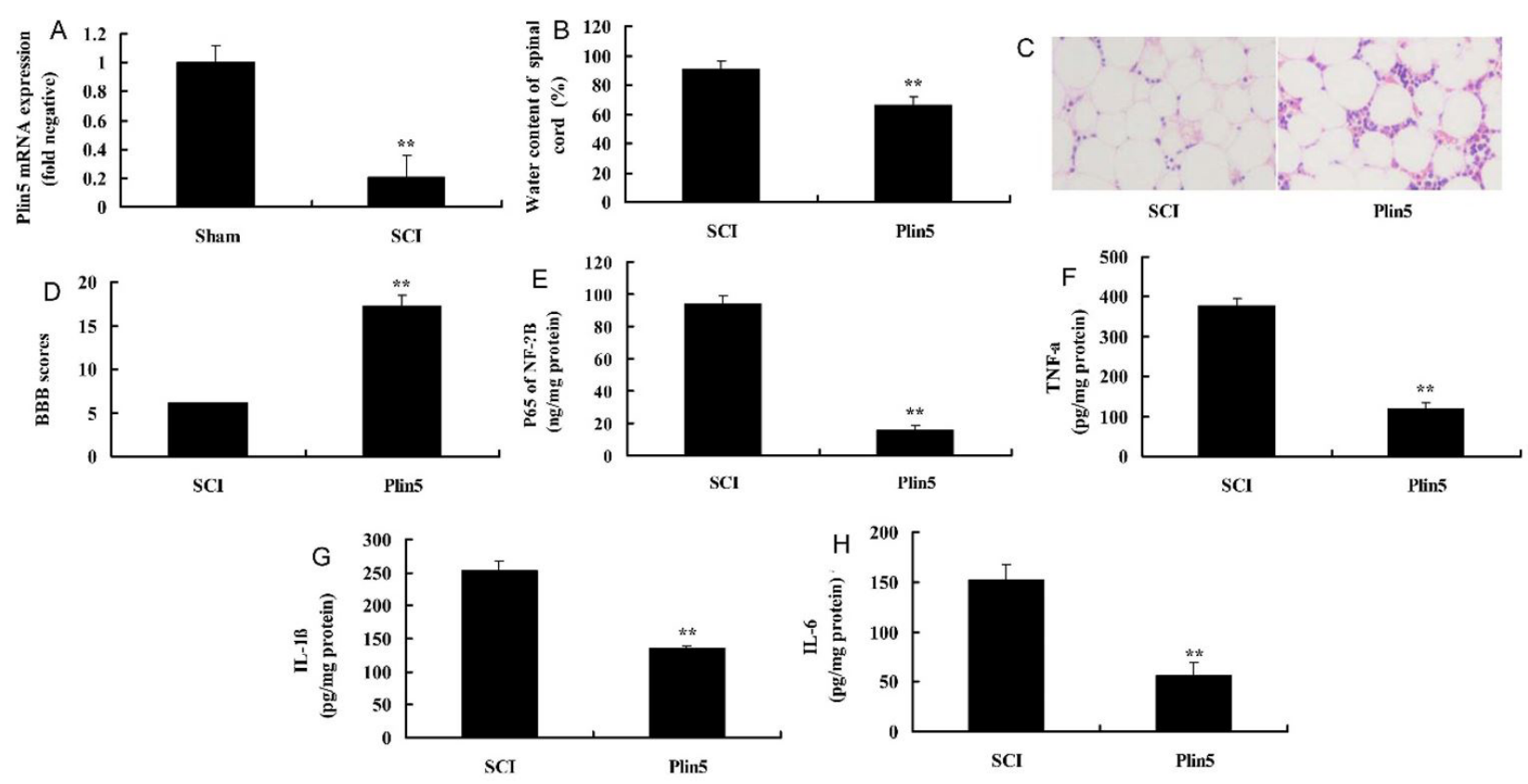

Figure 1. Serum levels of Plin5 in patient with SCI and Plin5 prevented SCI. Plin5 mRNA expression (A), the water content of spinal cord (B), Histological images (C), BBB scores (D), serum NF-KB p65 (E), TNF- $\alpha$ (F), IL-1 $\beta$ (G) and IL-6 (H) levels in mice of SCI. Sham, sham group; SCI, SCI model group; Plin5, SCI model mice by treatment with human Plin5 protein. ${ }^{\star *} \mathrm{p}<0.01$ compared with sham group.
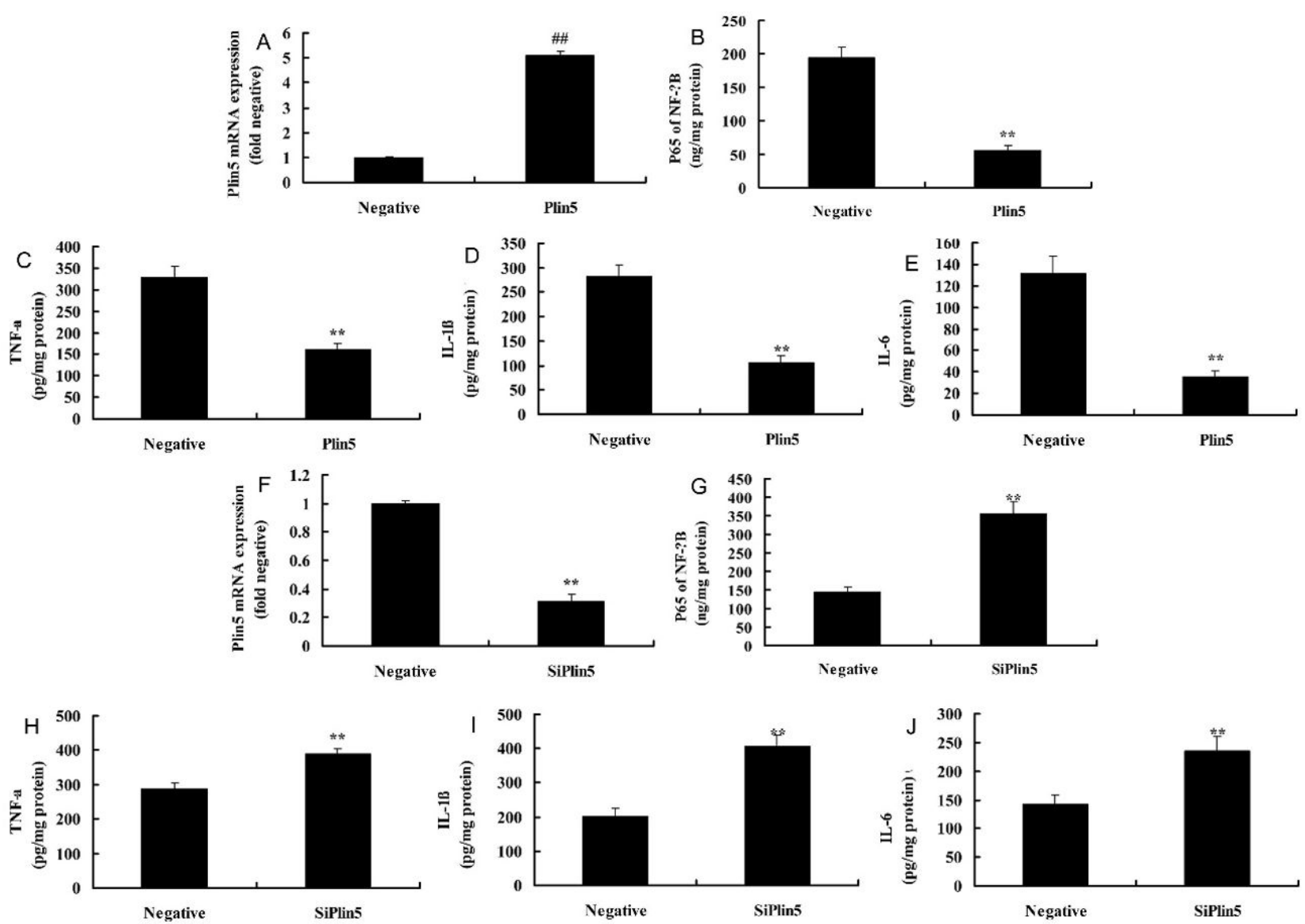

Figure 2. Plin 5 regulated inflammation of SCI. Plin5 mRNA expression (A), NF-KB p65 (B), TNF- $\alpha$ (C), IL-1 $\beta$ (D) and IL-6 (E) levels in vitro of SCI by over-expression of Plin5; Plin 5 mRNA expression (F), NF-KB p65 (G), TNF- $\alpha$ (H), IL-1 $\beta$ (I) and IL-6 (J) levels in vitro of SCI by down-regulation of Plin5. Negative, negative mimics group; Plin5, over-expression of Plin5 group; Si-Plin5, down-regulation of Plin5 group. ${ }^{* *} \mathrm{p}<0.01$ compared with negative mimics group. 

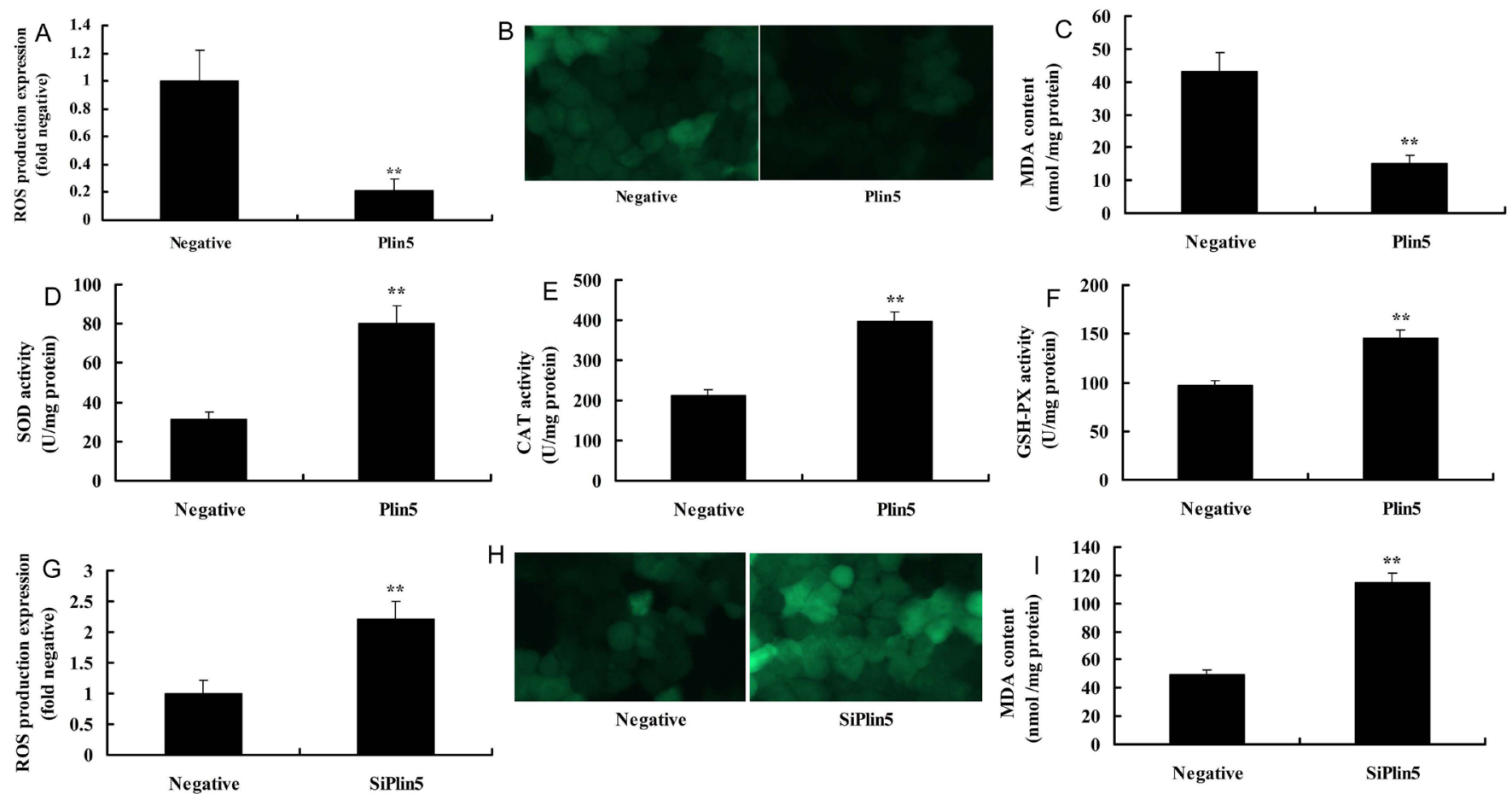

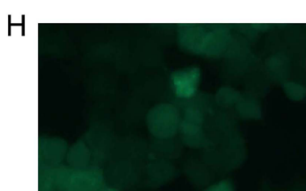

Negative

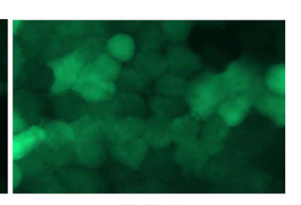

SiPlin5

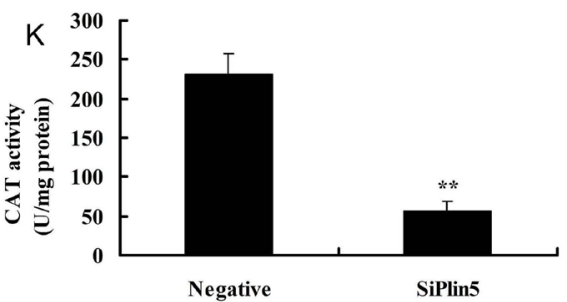

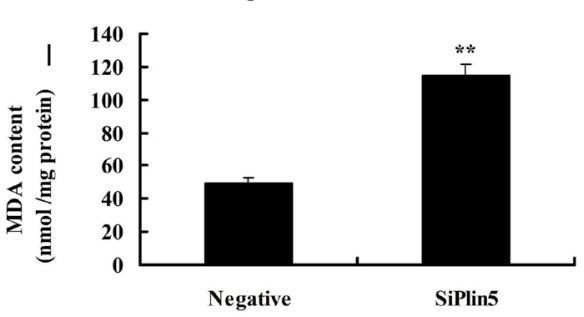

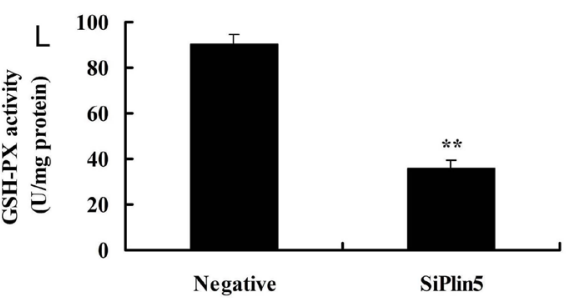

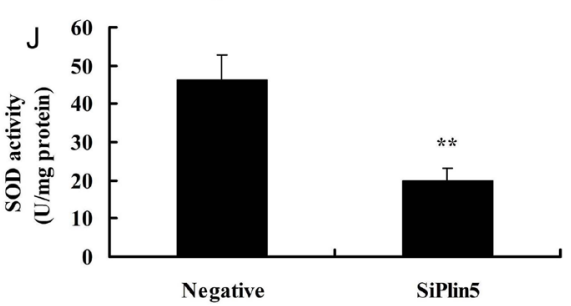

Figure 3. Plin5 regulated ROS-induced oxidative stress of SCI. ROS production levels (A and B), MDA activity level (C), SOD (D), CAT (E) and GSH-PX (F) activity levels in vitro of SCI by over-expression of Plin5; ROS production levels (G and H), MDA activity level (I), SOD (J), CAT $(\mathrm{K})$ and GSH-PX (L) activity levels in vitro of SCI by down-regulation of Plin5. Negative, negative mimics group; Plin5, over-expression of Plin5 group; Si-Plin5, down-regulation of Plin5 group. ${ }^{* *} \mathrm{p}<0.01$ compared with negative mimics group.

in vitro model of SCI (Figure 4C-4E). Down-regulation of Plin5 suppressed SIRT1 protein expression and induced NLRP3 protein expression in vitro model of SCI (Figure 4F-4H).

\subsection{The inhibition of SIRT1 reduced the effects of Plin5 on inflammation of SCI}

To further determine the role of SIRT1 in the effects of Plin5 on inflammation of SCI, siSIRT1 was used to down-regulated SIRT1 expression in SCI by over-expression of Plin5. SiSIRT1 suppressed SIRT1 protein expression and induced NLRP3 protein expression in vitro model of SCI by over-expression of Plin5, compared with over-expression of over-expression of Plin5 group (Figure 5A-5C). The inhibition of SIRT1 reduced the anti-inflammation effects of Plin 5 on NF-KB p65, TNF- $\alpha$, IL- $1 \beta$ and IL- 6 levels in vitro model SCI (Figure 5D-5G). The inhibition of SIRT1 also reduced the anti-oxidation effects of Plin5 on ROS production levels and MDA activity level, and SOD, CAT and GSH-PX activity levels in vitro model of SCI (Figure $5 \mathrm{H}-5 \mathrm{M}$ ).

\subsection{The activation of NLRP3 reduced the effects of Plin5 on inflammation of SCI}

To further confirm the role of NLRP 3 in the effects of Plin 5 on inflammation of SCI, NLRP3 plasmid induced NLRP3 protein expression of Plin5 on inflammation of SCI (Figure 6A-6B). The activation of NLRP3 increased NF-KB p65, TNF- $\alpha$, IL- $1 \beta$ and IL-6 levels in vitro model SCI by over-expression of Plin5, compared with over-expression of Plin5 group (Figure 6C-6F).

\section{Discussion}

SCI is a disorder of the central nervous system mainly characterized by functional disorder or deletion after direct or indirect violence (Gao et al., 2020). The inflammatory response caused by SCI has the dual effects of nerve damage and nerve protection (Hosseinzadeh et al., 2020; Hu et al., 2020; Li et al., 2020). Therefore, it is necessary to use its positive and negative roles in the repairing SCI, that is, selectively exerting its repair potential, while minimizing its destruction. We found that Plin5 mRNA expression was down-regulated in SCI mice, and 

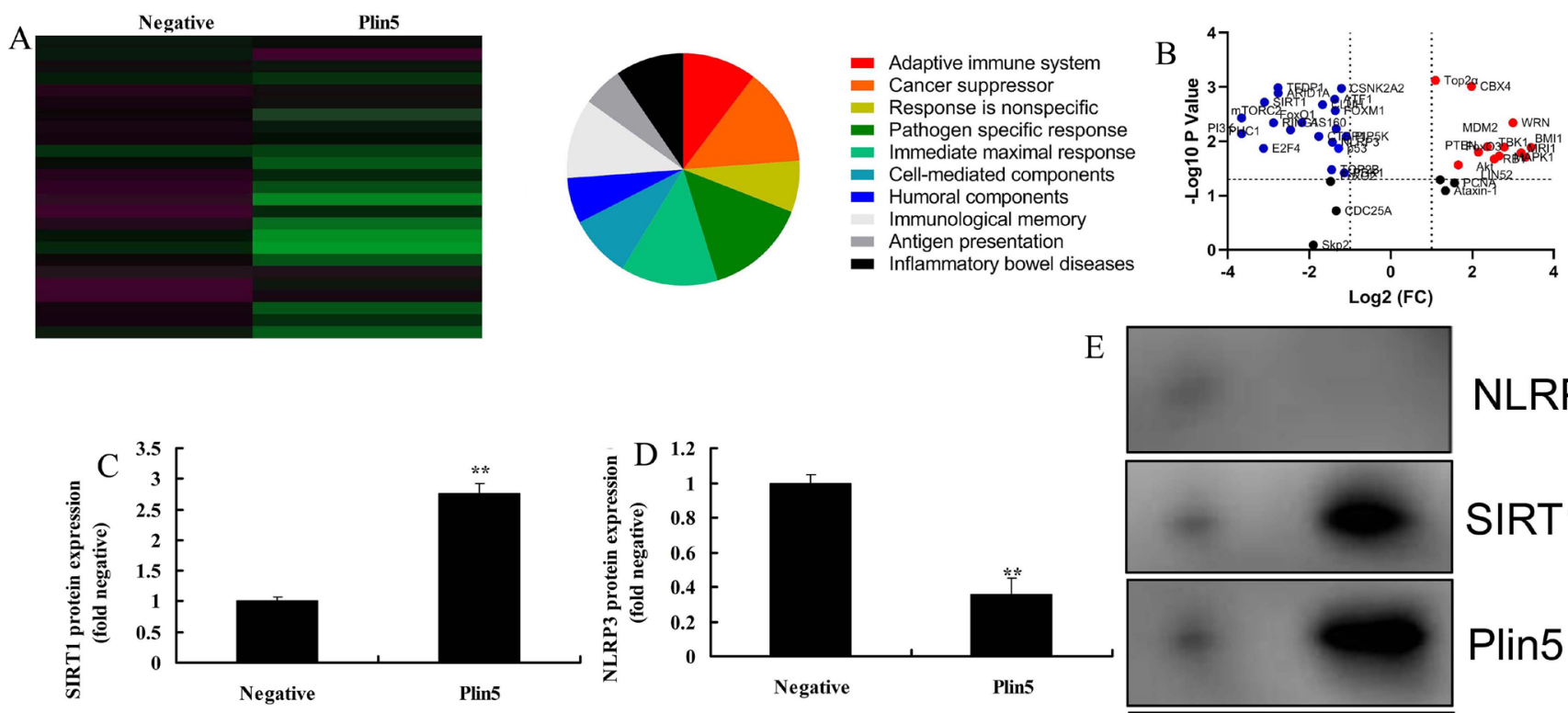

$\mathrm{E}$
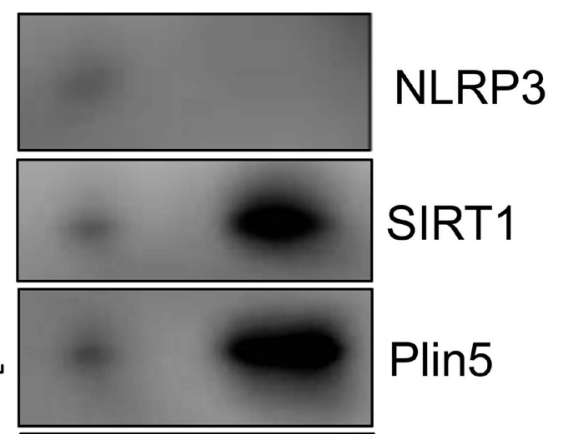

SIRT1

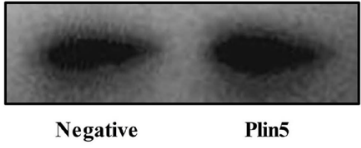

GAPDH

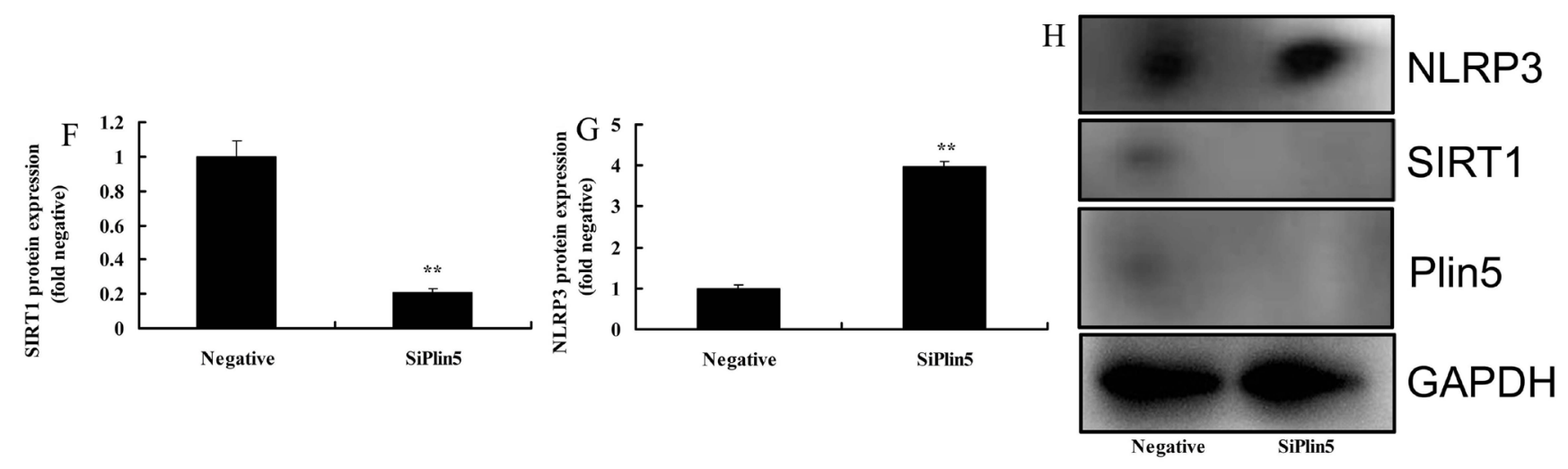

Figure 4. SIRT1 is control targets of anti-inflammation effects of Plin 5 in SCI by NLRP3. The results of gene chip (A), volcano figure (B), SIRT1 and NLRP3 protein expressions in vitro of SCI by over-expression of Plin5 (C, D and E); SIRT1 and NLRP3 protein expressions in vitro of SCI by down-regulation of Plin5 (F, G and H). Negative, negative mimics group; Plin5, over-expression of Plin5 group; Si-Plin5, down-regulation of Plin5 group. ${ }^{* *} \mathrm{p}<0.01$ compared with negative mimics group.

human Plin5 protein reduced the water content of spinal cord, increased BBB score and inhibited serum NF-KB p65, TNF- $\alpha$, IL- $1 \beta$ and IL- 6 levels. Zhou et al. supported that Plin 5 deficiency promotes atherosclerosis progression via Inflammation and oxidative stress (Zhou et al., 2017).

SCI is one of the main causes for disability, and its pathophysiological process includes primary injury and secondary injury (Yu et al., 2019a). Secondary SCI would initially recruit a large number of surrounding inflammatory cells can cause strong inflammatory responses, thereby leading to glial cell death (Zhao et al., 2017). The substrates of SIRT1 deacetylation mainly include FOXO1/3/4, c-myc, NF- $\kappa B$, etc (Zhao et al., 2017). By interacting with different substrates, SIRT1 exerts diverse biological functions, including regulating cell cycle, inhibiting apoptosis, protecting nerves, inhibiting inflammatory response, resisting oxidative stress, promoting metabolism of sugar and lipid, promoting neuronal proliferation and differentiation and participating autophagy process (Yu et al., 2019b). In the experimental study of inflammatory response, the research on SIRT1 is relatively in-depth. SIRT1, a NAD ${ }^{+}$-dependent protein deacetylase, exerts an obvious anti-inflammatory effect (Yu et al., 2019b). This study showed that Plin5 reduced inflammation and ROS-induced oxidative stress of SCI by induction of SIRT1 expression. Zhang et al. (2020a) show that hepatic PLIN5 promote autophagy and prevent inflammation via SIRT1 signals.

Apart from various neurological diseases, NLRP3 is also closely associated with the development of diabetes, atherosclerosis, gout, etc. This experiment can confirm that in acute SCI, NLRP3 inflammasome is also involved in the inflammatory response after acute SCI. Studies have all shown 

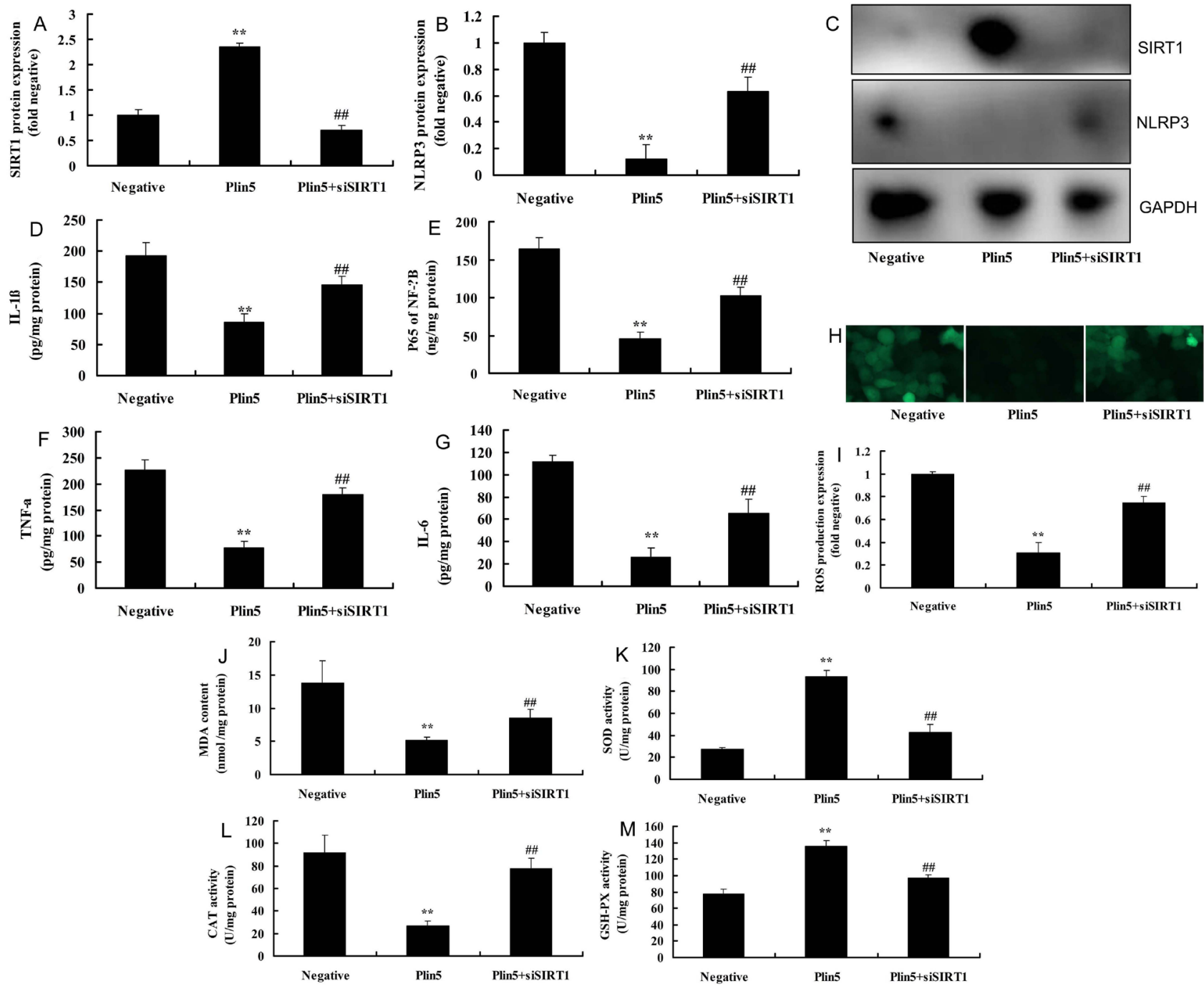

Figure 5. The inhibition of SIRT1 reduced the effects of Plin 5 on inflammation of SCI. SIRT1 and NLRP3 protein expressions (A, B, C), NF-KB p65 (D), TNF- $\alpha$ (E), IL-1 $\beta$ (F), IL-6 (G), ROS production levels (H and I), MDA activity level (J), SOD (K), CAT (L) and GSH-PX (M) activity levels. Negative, negative mimics group; Plin5, over-expression of Plin5 group; Plin5+siSIRT1, over-expression of Plin5 group and down-regulation of SIRT1 group. ${ }^{* *} \mathrm{p}<0.01$ compared with negative mimics group, $\#$ $\mathrm{p}<0.01$ compared with over-expression of Plin5 group.
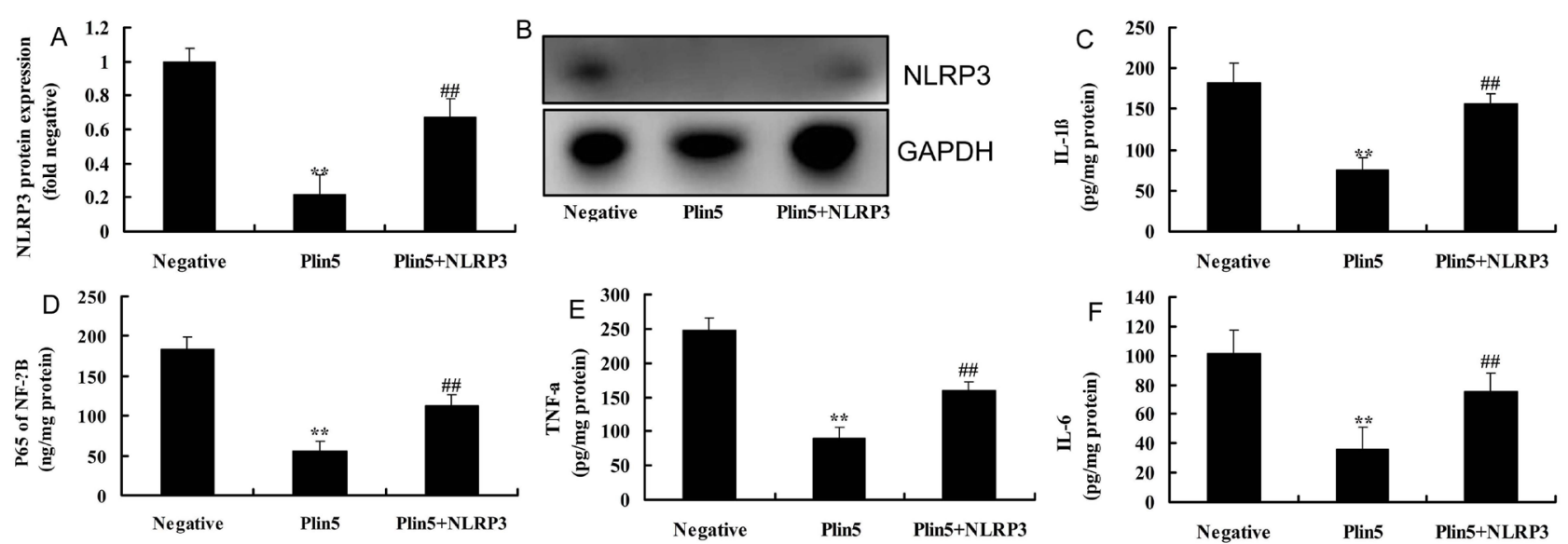

Figure 6. The activation of NLRP3 reduced the effects of Plin5 on inflammation of SCI. NLRP3 protein expressions (A and B), NF-KB p65 (C), TNF- $\alpha$ (D), IL-1 $\beta$ (E), IL-6 (F). Negative, negative mimics group; Plin5, over-expression of Plin5 group; Plin5+ NLRP3, over-expression of Plin5 group and over-expression of NLRP3 group. ${ }^{* *} \mathrm{p}<0.01$ compared with negative mimics group, \#\#p $<0.01$ compared with over-expression of Plin5 group. 
that NLRP3 inflammasome is an important factor for secondary SCI (Liu et al., 2020; Jiao et al., 2020). These results of this study showed that the activation of NLRP3 reduced the effects of Plin5 on inflammation of SCI. Asimakopoulou et al. conclude that PLIN5 protects sgainst hepatic injury via inflammasome activation (Asimakopoulou et al., 2020).

In summary, Plin 5 mRNA expression was down-regulated in SCI mice, and Plin5 prevent SCI and related to inflammation. Plin5 reduced inflammation and ROS-induced oxidative stress of SCI by induction of SIRT1/NLRP3 signal. The findings of the present study provided further mechanistic support for Plin5 as a promising treatment with SCI agent.

\section{References}

Asimakopoulou, A., Engel, K. M., Gassler, N., Bracht, T., Sitek, B., Buhl, E. M., Kalampoka, S., Pinoé-Schmidt, M., van Helden, J., Schiller, J., \& Weiskirchen, R. (2020). Deletion of Perilipin 5 protects against hepatic injury in nonalcoholic fatty liver disease via missing inflammasome activation. Cells, 9(6), 1346. http://dx.doi.org/10.3390/ cells9061346. PMid:32481590.

Ding, H., Shi, Z., Zhang, L., Gao, N., Cheng, X., Lin, H., Zhang, Z., \& Zhang, G. (2021). Progranulin (PGRN) is serves as an inflammationresponse biomarker and promotes lung damage in burn-induced Sepsis via the SIRT1 Pathways by ROS. Food Science and Technology. In press. https://doi.org/10.1590/fst.36720.

Gao, K., Niu, J., \& Dang, X. (2020). Neuroprotection of melatonin on spinal cord injury by activating autophagy and inhibiting apoptosis via SIRT1/AMPK signaling pathway. Biotechnology Letters, 42(10), 2059-2069. http://dx.doi.org/10.1007/s10529-020-02939-5. PMid:32514788.

Gensel, J. C., \& Zhang, B. (2015). Macrophage activation and its role in repair and pathology after spinal cord injury. Brain Research, 1619, 1-11. http://dx.doi.org/10.1016/j.brainres.2014.12.045. PMid:25578260.

Hoey, R. F., \& Hubscher, C. H. (2020). Investigation of bowel function with anorectal manometry in a rat spinal cord contusion model. Journal of Neurotrauma, 37(18), 1971-1982. http://dx.doi.org/10.1089/ neu.2020.7145. PMid:32515264.

Hosseinzadeh, S., Lindsay, S. L., Gallagher, A. G., Wellings, D. A., Riehle, M. O., Riddell, J. S., \& Barnett, S. C. (2020). A novel poly-e-lysine based implant, Proliferate ${ }^{\oplus}$, for promotion of CNS repair following spinal cord injury. Biomaterials Science, 8(13), 3611-3627. http:// dx.doi.org/10.1039/D0BM00097C. PMid:32515439.

Hu, X., Li, R., Wu, Y., Li, Y., Zhong, X., Zhang, G., Kang, Y., Liu, S., Xie, L., Ye, J., \& Xiao, J. (2020). Thermosensitive heparin-poloxamer hydrogel encapsulated bFGF and NGF to treat spinal cord injury. Journal of Cellular and Molecular Medicine, 24(14), 8166-8178. http:// dx.doi.org/10.1111/jcmm.15478. PMid:32515141.

Järve, A., Qadri, F., Todiras, M., Schmolke, S., \& Bader, M. (2020). Angiotensin-II receptor type Ia does not contribute to cardiac atrophy following high-thoracic spinal cord injury in mice. Experimental Physiology, 105(8), 1316-1325. http://dx.doi.org/10.1113/EP088378. PMid:32515106.

Jiang, W., Li, M., He, F., \& Zhu, L. (2019). Inhibition of NLRP3 inflammasome attenuates spinal cord injury-induced lung injury in mice. Journal of Cellular Physiology, 234(5), 6012-6022. http:// dx.doi.org/10.1002/jcp.27233. PMid:30589073.

Jiao, J., Zhao, G., Wang, Y., Ren, P., \& Wu, M. (2020). MCC950, a selective inhibitor of NLRP3 inflammasome, reduces the inflammatory response and improves neurological outcomes in mice model of spinal cord injury. Frontiers in Molecular Biosciences, 7, 37. http:// dx.doi.org/10.3389/fmolb.2020.00037. PMid:32195267.

Kjell, J., \& Olson, L. (2016). Rat models of spinal cord injury: from pathology to potential therapies. Disease Models \& Mechanisms, 9(10), 1125-1137. http://dx.doi.org/10.1242/dmm.025833. PMid:27736748.

Kornhaber, R., Mclean, L., Betihavas, V., \& Cleary, M. (2018). Resilience and the rehabilitation of adult spinal cord injury survivors: a qualitative systematic review. Journal of Advanced Nursing, 74(1), 23-33. http:// dx.doi.org/10.1111/jan.13396. PMid:28726274.

Li, S., Alam, M., Ahmed, R. U., Zhong, H., Wang, X. Y., Ng, S., \& Zheng, Y. P. (2020). Ultrasound-driven piezoelectric current activates spinal cord neurocircuits and restores locomotion in rats with spinal cord injury. Bioelectronic Medicine, 6(1), 13. http://dx.doi.org/10.1186/ s42234-020-00048-2. PMid:32514413.

Liu, Z., Yao, X., Jiang, W., Li, W., Zhu, S., Liao, C., Zou, L., Ding, R., \& Chen, J. (2020). Advanced oxidation protein products induce microglia-mediated neuroinflammation via MAPKs-NF- $\kappa B$ signaling pathway and pyroptosis after secondary spinal cord injury. Journal of Neuroinflammation, 17(1), 90. http://dx.doi.org/10.1186/s12974020-01751-2. PMid:32192500.

Lu, P., Han, D., Zhu, K., Jin, M., Mei, X., \& Lu, H. (2019). Effects of Sirtuin 1 on microglia in spinal cord injury: involvement of Wnt $/ \beta$ catenin signaling pathway. Neuroreport, 30(13), 867-874. http:// dx.doi.org/10.1097/WNR.0000000000001293. PMid:31373965.

Ma, Z., Lu, Y., Yang, F., Li, S., He, X., Gao, Y., Zhang, G., Ren, E., Wang, Y., \& Kang, X. (2020). Rosmarinic acid exerts a neuroprotective effect on spinal cord injury by suppressing oxidative stress and inflammation via modulating the Nrf2/HO- 1 and TLR4/NF- $\kappa \mathrm{B}$ pathways. Toxicology and Applied Pharmacology, 397, 115014. http:// dx.doi.org/10.1016/j.taap.2020.115014. PMid:32320792.

Mohammed, I., Ijaz, S., Mokhtari, T., Gholaminejhad, M., Mahdavipour, M., Jameie, B., Akbari, M., \& Hassanzadeh, G. (2020). Subventricular zone-derived extracellular vesicles promote functional recovery in rat model of spinal cord injury by inhibition of NLRP3 inflammasome complex formation. Metabolic Brain Disease, 35(5), 809-818. http:// dx.doi.org/10.1007/s11011-020-00563-w. PMid:32185593.

Pu, Z., Han, C., Zhang, W., Xu, M., Wu, Z., Liu, Y., Wu, M., Sun, H., \& Xie, H. (2019). Systematic understanding of the mechanism and effects of Arctigenin attenuates inflammation in dextran sulfate sodium-induced acute colitis through suppression of NLRP3 inflammasome by SIRT1. American Journal of Translational Research, 11(7), 3992-4009. PMid:31396314.

Su, X. Q., Wang, X. Y., Gong, F. T., Feng, M., Bai, J. J., Zhang, R. R., \& Dang, X. Q. (2020). Oral treatment with glycyrrhizin inhibits NLRP3 inflammasome activation and promotes microglial $\mathrm{M} 2$ polarization after traumatic spinal cord injury. Brain Research Bulletin, 158, 1-8. http://dx.doi.org/10.1016/j.brainresbull.2020.02.009. PMid:32092434.

Wang, D., Zhang, Z., Si, Z., \& Wang, L. (2021). Circ 0006282/miR-155 reduced inflammation in diabetic nephropathy via expression of SIRT1/NLRP3 signaling pathway. Food Science and Technology. In press. https://doi.org/10.1590/fst.39520.

Wang, X., Su, X., Gong, F., Yin, J., Sun, Q., Lv, Z., \& Liu, B. (2019). MicroRNA-30c abrogation protects against spinal cord ischemia reperfusion injury through modulating SIRT1. European Journal of Pharmacology, 851, 80-87. http://dx.doi.org/10.1016/j.ejphar.2019.02.027. PMid:30776367.

Wang, Y., Pang, Q. J., Liu, J. T., Wu, H. H., \& Tao, D. Y. (2018). Downregulated miR-448 relieves spinal cord ischemia/reperfusion injury by up-regulating SIRT1. Brazilian Journal of Medical and Biological Research, 51(5), e7319. http://dx.doi.org/10.1590/1414-431x20177319. PMid:29561961. 
Yu, L., \& Qian, J. (2020). Dihydrotanshinone I Alleviates Spinal Cord Injury via Suppressing Inflammatory Response, Oxidative Stress and Apoptosis in Rats. Medical Science Monitor, 26, e920738. http:// dx.doi.org/10.12659/MSM.920738. PMid:32112706.

Yu, S., Xie, L., Liu, Z., Li, C., \& Liang, Y. (2019a). MLN4924 exerts a neuroprotective effect against oxidative stress via Sirt1 in spinal cord ischemia-reperfusion injury. Oxidative Medicine and Cellular Longevity, 2019, 7283639. http://dx.doi.org/10.1155/2019/7283639. PMid:31178972.

Yu, X., Zhang, S., Zhao, D., Zhang, X., Xia, C., Wang, T., Zhang, M., Liu, T., Huang, W., \& Wu, B. (2019b). SIRT1 inhibits apoptosis in in vivo and in vitro models of spinal cord injury via microRNA-494. International Journal of Molecular Medicine, 43(4), 1758-1768. http:// dx.doi.org/10.3892/ijmm.2019.4106. PMid:30816451.

Zhang, E., Cui, W., Lopresti, M., Mashek, M. T., Najt, C. P., Hu, H., \& Mashek, D. G. (2020a). Hepatic PLIN5 signals via SIRT1 to promote autophagy and prevent inflammation during fasting. Journal of Lipid
Research, 61(3), 338-350. http://dx.doi.org/10.1194/jlr.RA119000336. PMid:31932301.

Zhang, M., Wang, L., Huang, S., \& He, X. (2020b). MicroRNA-223 targets NLRP3 to relieve inflammation and alleviate spinal cord injury. Life Sciences, 254, 117796. http://dx.doi.org/10.1016/j.lfs.2020.117796. PMid:32417375.

Zhao, H., Chen, S., Gao, K., Zhou, Z., Wang, C., Shen, Z., Guo, Y., Li, Z., Wan, Z., Liu, C., \& Mei, X. (2017). Resveratrol protects against spinal cord injury by activating autophagy and inhibiting apoptosis mediated by the SIRT1/AMPK signaling pathway. Neuroscience, 348, 241-251. http://dx.doi.org/10.1016/j.neuroscience.2017.02.027. PMid:28238848.

Zhou, P. L., Li, M., Han, X. W., Bi, Y. H., Zhang, W. G., Wu, Z. Y., \& Wu, G. (2017). Plin5 deficiency promotes atherosclerosis progression through accelerating inflammation, apoptosis and oxidative stress. Journal of Cellular Biochemistry. In press. PMid:29215758. 